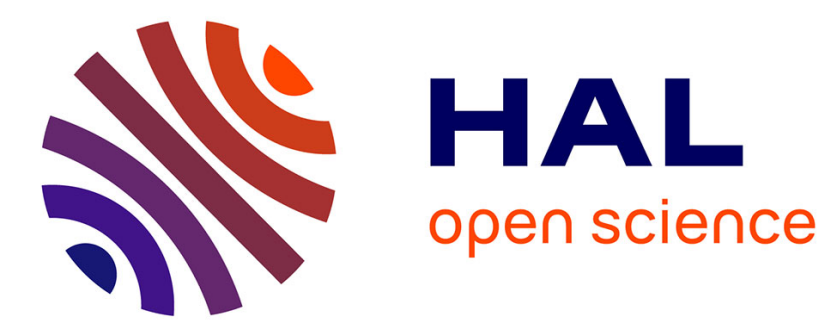

\title{
Utilisation du détecteur courbe CPS 120 INEL en position horizontale ou verticale. Application à l'étude des composés d'insertion graphite-lithium
}

\author{
M. Lelaurain, F. Henry, J. Roux, G. Medjahdi
}

\section{- To cite this version:}

M. Lelaurain, F. Henry, J. Roux, G. Medjahdi. Utilisation du détecteur courbe CPS 120 INEL en position horizontale ou verticale. Application à l'étude des composés d'insertion graphite-lithium. Journal de Physique IV Proceedings, 1996, 06 (C4), pp.C4-907-C4-912. 10.1051/jp4:1996487 . jpa00254370

\author{
HAL Id: jpa-00254370 \\ https://hal.science/jpa-00254370
}

Submitted on 1 Jan 1996

HAL is a multi-disciplinary open access archive for the deposit and dissemination of scientific research documents, whether they are published or not. The documents may come from teaching and research institutions in France or abroad, or from public or private research centers.
L'archive ouverte pluridisciplinaire HAL, est destinée au dépôt et à la diffusion de documents scientifiques de niveau recherche, publiés ou non, émanant des établissements d'enseignement et de recherche français ou étrangers, des laboratoires publics ou privés. 


\title{
Utilisation du détecteur courbe CPS 120 INEL en position horizontale ou verticale. Application à l'étude des composés d'insertion graphite-lithium
}

\author{
M. Lelaurain, F.X. Henry, J.P. Roux* et G. Medjahdi \\ LCMA, Université Nancy I, BP. 239, 54506 Vandouvre cedex, France \\ * LEMD, CNRS, 25 avenue des Martyrs, 38000 Grenoble, France
}

\begin{abstract}
Résumé: En utilisant les propriétés texturales des matériaux lamellaires ou fibrillaires hautement orientés, nous pouvons étudier "in-situ" la structure de composés, au cours de leur insertion, selon la méthode du "cristal tournant". Pour cela, nous avons réalisé un diffractomètre muni d'un détecteur courbe (CPS 120 INEL), que l'on peut placer horizontalement ou verticalement de manière à envelopper la sphère d'Ewald pour l'enregistrement des rangées (hkl)* que l'on souhaite étudier, L'échantillon est fixe; le montage, par transmission. Ce dispositif nous a permis de mettre en évidence la modification de l'empilement des feuillets de graphène dans les composés d'insertion graphite-lithium de deuxième stade et de complèter la caractérisation structurale de ces composés.
\end{abstract}

\begin{abstract}
The textural properties of lamellar or fibrillar highly oriented materials permit studying "in-situ" the structure changes which accompany intercalation into them, using the "rotating-crystal" method. To do so, we have used a diffractometer equipped with an INEL CPS 120 detector which can be placed in either a horizontal or vertical position, so as to completely encompass the Ewald sphere and allow recording the (hkl)* rods at any given point of reciprocal space. The sample is fixed in position and we used a transmission setup. We show the experimental diagrams corresponding to the graphene stacking changes in second stage graphite-lithium intercalation compounds which have allowed completing the structural characterization of these phases.
\end{abstract}

\section{INTRODUCTION}

Les matériaux lamellaires ou fibrillaires tels que les composés d'insertion du graphite ou les polymères électroactifs, doivent faire l'objet d'une caractérisation structurale très fine préalablement à l'étude de leurs propriétés physiques. Cette étude structurale passe par l'enregistrement des distributions d'intensité le long de rangées cristallographiques choisies afin d'accèder à la nature de certains défauts et à l'ordre d'empilement du réactif intercalé par rapport à la matrice. Mais certains composés sont très altérables à l'air ou doivent être étudiés "in-situ" ou dans des porte-échantillons étanches qui ne permettent pas de les orienter commodément au centre des dispositifs goniométriques classiques. Aussi, pour les étudier, doit-on concevoir des montages qui allient souplesse d'utilisation et possibilités de traitement des résultats. Sur le principe du "cristal tournant", grâce aux propriétés des matériaux hautement orientés, nous avons mis au point un diffractomètre qui utilise un détecteur courbe, placé horizontalement ou verticalement selon l'orientation de l'échantillon par rapport à la direction du faisceau incident et les rangées de l'espace réciproque que l'on souhaite enregistrer.

Ce dispositif a déjà servi à déterminer l'organisation tridimensionnelle des composés polyacétylène-métaux alcalins lourds [1]. Nous présenterons, ici, les résultats expérimentaux 
relatifs à l'étude des composés d'insertion du graphite avec le lithium, préparés par voie électrochimique [2], après un bref rappel de la structure des matériaux et la présentation du diffractomètre que nous avons construit: principe, fonctionnement et caractéristiques.

\section{STRUCTURE DES MATERIAUX CARBONES}

\subsection{Le graphite}

Matériau lamellaire, le graphite, sous sa forme la plus stable, possède une structure hexagonale [3]. Les atomes de carbone sont disposés selon un pavage hexagonal $\left(\mathrm{d}_{\mathrm{C}-\mathrm{C}}=142 \mathrm{pm}\right)$ dans les plans de graphène distants de 335 pm et translatés les uns des autres d'un vecteur $\pm \vec{\Delta}=\vec{a}_{1} / 3+$ $2 \overrightarrow{\mathrm{a}}_{2} / 3$ pour former un empilement de type $\mathrm{ABAB}$....(fig1).

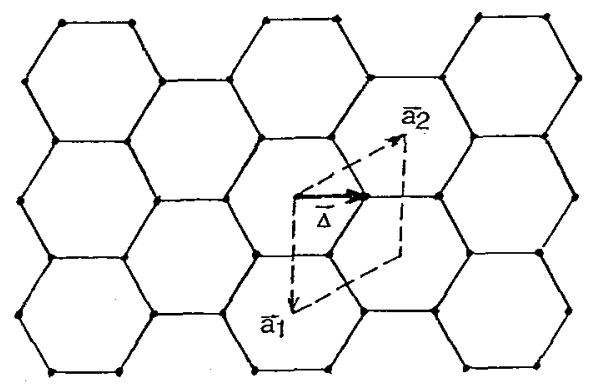

Figure 1: Position des atomes de carbone dans un feuillet de graphène et définition du vecteur de translation [4]

\subsection{Le Pyrographite}

S'il est hautement orienté (H.O.P.G.), le pyrographite peut être décrit comme un ensemble de cristallites dont les axes $\vec{c}$ sont quasi-parallèles, alors que les axes $\vec{a}$ se répartissent statistiquement dans le plan perpendiculaire à $\vec{c}$. Le pyrographite que nous avons utilisé (PGCCL Carbone-Lorraine) possède une mosaïcité $\leq 1^{\circ}$. Matériaux de choix pour l'étude des propriétés physiques des composés d'insertion du graphite, cette "poudre" hautement orientée, va grandement faciliter les études structurales car elle permettra l'examen successif des réflexions 001 , hk0 et hkl, selon la disposition des échantillons par rapport au faisceau incident [5-6].

\subsection{Les composés d'insertion}

Le caractère amphotère du graphite, lui permet de réagir avec des donneurs ou des accepteurs d'électrons pour former des composés d'insertion dont la particularité est de posséder un arrangement périodique de l'insérat à longue distance dans le réseau hôte. Au cours de l'insertion, les feuillets élémentaires de carbone restent pratiquement intacts; ils s'écartent pour laisser place au réactif et glissent dans leur propre plan. On caractérisera un composé d'insertion par son stade $\mathbf{n}$ (nombre de couches de graphène entre deux plans d'insérat), sa période d'identité $I_{C}$ qui correspond à la distance qui sépare deux couches d'insérat consécutives et la distance interplanaire $\boldsymbol{d}_{\mathbf{i}}$ séparant deux plans de graphène intercalés. Généralement,

$$
I_{c}=d_{i}+(n-1) 335 p m \text {. }
$$

Les séquences d'empilement des feuillets carbonés qui, de part et d'autre de la couche insérée ( $)$, peuvent être de même type $(\mathrm{A} / \mathrm{A}, \mathrm{B} / \mathrm{B}, \ldots)$, partiellement ou totalement décalés tel que 
$(A / B, \ldots)$, sont généralement de type $(A B A B \ldots)$ lorsque les feuillets ne sont pas insérés; nous verrons qu'il n'en va pas de même pour les composés graphite-lithium de stade $n=2$.

Outre la détermination des paramètres précités, l'analyse structurale d'un composé devra mettre en évidence la dimensionnalité de la structure du réactif: $2 \mathrm{D}$ ou $3 \mathrm{D}$, et la commensurabilité ou non des deux sous réseaux (hôte et insérat).

\section{TECHNIQUES EXPERIMENTALES}

\subsection{Principes}

Comme nous l'avons déjà signalé, classiquement, l'étude des composés d'insertion préparés à base de pyrographite passe par l'examen successif des réflexions (00l) (hk0) et (hkl); néanmoins une visualisation de l'ensemble de l'espace réciproque est souvent très utile, car elle permet de mettre en évidence le degré d'ordre dans l'empilement des couches de carbone et la dimensionnalité de la structure du réactif inséré (2D ou 3D). Pour y parvenir, la méthode du "cristal tournant" est bien adaptée et simple à mettre en oeuvre.

La formation du diagramme est représentée par la construction d'Ewald. Les plans réciproques coupent la sphère suivant des cercles parallèles équidistants. Le lieu des rayons diffractés est une famille de cônes de révolution qui s'appuient sur ces cercles[7].

Dans le cas de matériaux lamellaires, $\overrightarrow{\mathfrak{c}}$ est l'axe de rotation et la strate équatoriale contient l'ensemble des réflexions $(\mathrm{hk} 0)^{*}$. Si les matériaux sont préparés à base de HOPG, toutes les orientations sont présentes sans qu'il soit nécessaire de faire tourner l'échantillon; à chaque réflexion (hk1)* correspond un tore centré sur l'axe $\vec{c}^{*}$, lorsque les réseaux sont $3 \mathrm{D}$ (fig2a), ou un cylindre lorsque l'ordre est $2 \mathrm{D}$ (fig2b).
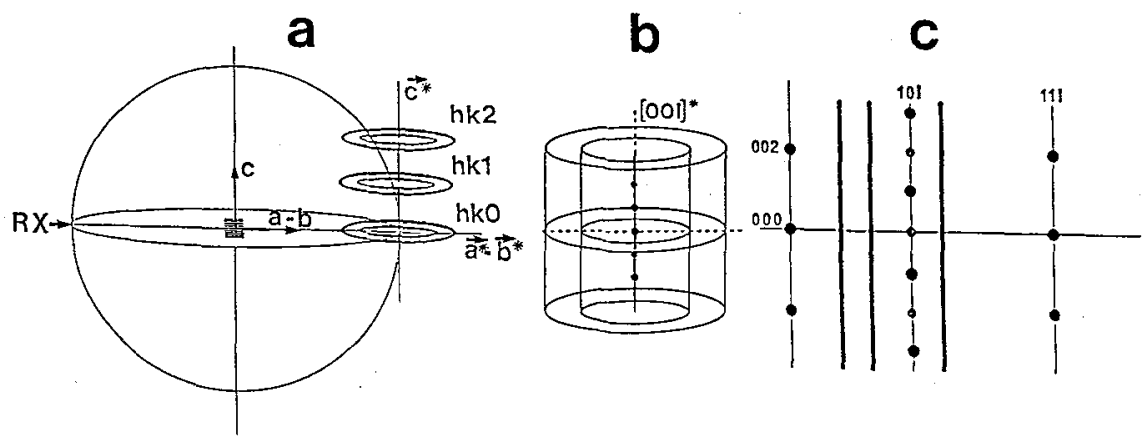

Figure 2: Intersection de l'espace réciproque d'une poudre orientée avec la sphère d'Ewald- a: ordre 3D, b: espace réciproque d'un matériau 2D ou turbostratique[7] c: image de diffraction de deux types de réseaux imbriqués: exemple d'un composé ou le graphite serait en $A / B$...et l'insérat $2 \mathrm{D}$.

Quand on utilise une courte longueur d'onde, comme celle du molybdène, l'information recueillie sur un simple film plan est suffisante, mais néanmoins déformée; si l'on est capable d'enregistrer les réflexions (hkl)* en enveloppant la sphère d'Ewald, l'exploitation des résultats en sera grandement facilitée. 


\subsection{Montage goniométrique}

L'ensemble de diffractométrie que nous avons construit, est schématisé (fig3). Il comporte

- un axe vertical (A) autour duquel peuvent tourner, grâce à un moteur pas-à-pas, un bras porte-détecteur et un plateau central qui permet aussi l'utilisation du dispositif dans les conditions classiques de diffractométrie sur poudre; la vitesse de rotation du plateau et du détecteur sont à choisir entre 1 et $60^{\circ} / \mathrm{mn}$, avec un pas de $5 / 1000^{\circ}$;

- le détecteur courbe INEL CPS 120, fixé en son centre sur un pivot (O) pour permettre son utilisation en position horizontale ou verticale $(+\theta /-\theta)$;

- des collimateurs d'entrée rétractables (C), de diamètre $\geq 5 / 10 \mathrm{~mm}$ sur le trajet du faisceau incident et un puits $(\mathrm{P})$ situé derrière le porte-échantillon.

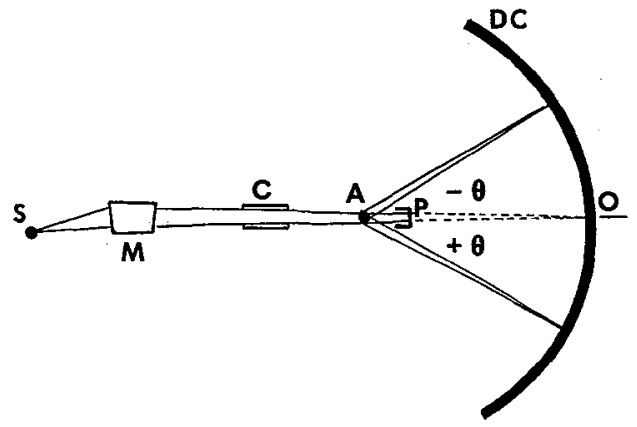

Figure 3: Schéma du montage goniométrique par transmission

L'ensemble est piloté par un micro-ordinateur, à l'aide d'un logiciel développé au laboratoire lequel, permet également le traitement des résultats.

Nous utilisons le rayonnement du molybdène provenant d'un générateur à anode tournante RIGAKU; le foyer est ponctuel (S); le faisceau monochromatique ((M)quartz) est focalisé sur le cercle du détecteur $(0)$, la distance de focalisation est de $500 \mathrm{~mm}$.

Dans ces conditions, les raies de diffraction d'une poudre de diamant, que nous utilisons le plus souvent comme référence pour l'ensemble de nos mesures, ont pour largeur à mi-hauteur $\Delta \theta=$ $0.062^{\circ} \pm 0.002$, lorsque le détecteur est en position horizontale et $0.10^{\circ} \pm 0.01$, en position verticale; cette dernière valeur pourrait être améliorée par l'emploi d'un monochromateur double courbure .

\section{APPLICATION A L'ETUDE DES COMPOSES GRAPHITE-LI DE STADE 2}

La structure, à la température ambiante, des composés graphite-lithium de stade 1 et 2 a, pour l'essentiel, été définie au début des années 70 [8] et l'existence, pour les composés de deuxième stade, de deux types d'organisation structurale en fonction de la composition des couches insérées, a depuis été confirmée [9-10]. Cependant, la caractérisation de ces deux phases est restée incomplète car les modes de préparation utilisés par les différents auteurs, n'avaient pas permis de les isoler totalement. Depuis, l'insertion du lithium par voie électrochimique a été maîtrisée [2], elle nous a permis d'obtenir des phases pures et de préciser la composition et la structure des composés dits "riches" $\mathrm{LiC}_{12}$ et "pauvres" $\mathrm{LiC}_{18}$. 


\subsection{Mise en place des échantillons et mesures}

Pour cette étude, les composés, après insertion ont pu êtres transférés, sous atmosphère inerte, dans des tubes en verre de Lindemann. Ce sont des bâtonnets rectangulaires de petite dimensions $(10 \times 1 \times 0.5 \mathrm{~mm})$. On les dispose sur une tête goniométrique de sorte que le faisceau incident les traverse dans la largeur et que l'axe $\vec{c}$ soit vertical.

Dans un premier temps, on enregistre la strate équatoriale, le détecteur placé horizontalement; ensuite, chaque reflexion (hk0) sera l'origine de la rangée (hkl) que l'on souhaite étudier. Le détecteur est alors positionné verticalement et déplacé de l'angle $\theta$ désiré.

\subsection{Résultats}

Nous ne présenterons ici, que quelques résultats expérimentaux d'une étude par ailleurs publiée [11] qui illustrent les possibilités du goniomètre qui vient d'être décrit.
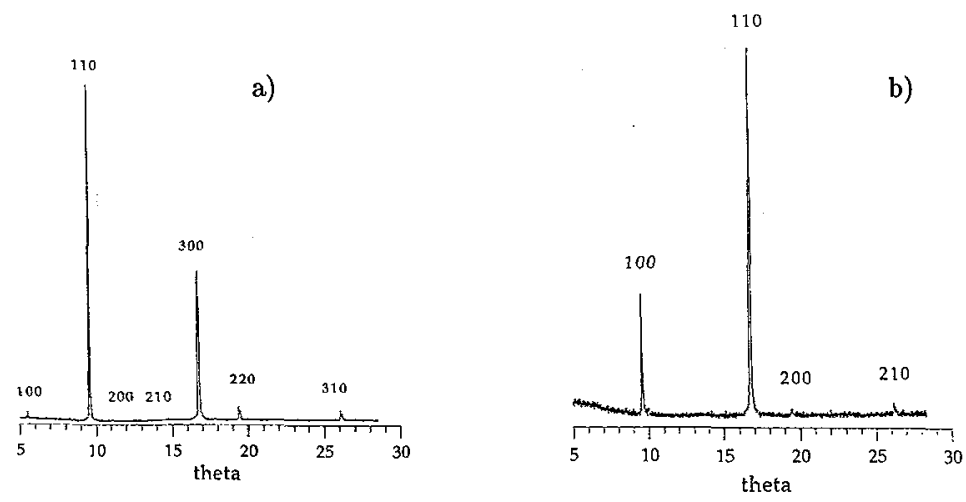

Figure 4: Réflexions (hk0) des composés graphite-lithium de stade 2, a: $\mathrm{LiC}_{12}$, b: $\mathrm{Lic}_{18}$.

(a)

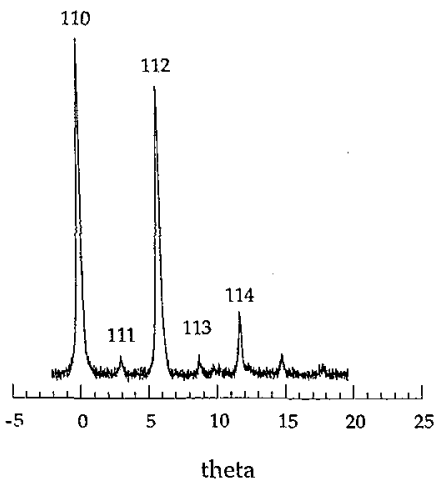

(b)

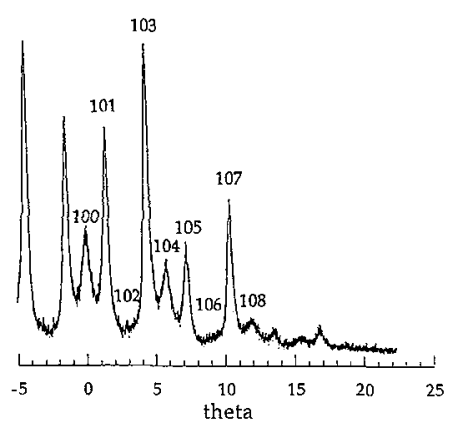

Figure 5: Réflexions (10l) du sous réseau carboné dans lcs composés graphite-Li de stade 2 a: $\mathrm{LiC}_{12}$, b: $\mathrm{LiC}_{18}$ 
Sur les figures $4 a$ et $4 b$, nous avons reproduit les enregistrements des réflexions (hk0) des composés $\mathrm{LiC}_{12}$ et $\mathrm{LiC}_{18}$. On peut constater que les raies du sous-réseau de lithium n'apparaissent que pour $\mathrm{LiC}_{12}$ et que les intensités des réflexions correspondant au réseau carboné n'ont pas la même intensité. En fait dans le stade 2 "riche" les atomes de lithium sont disposés de manière périodique au centre des hexagones de graphite selon un réseau hexagonal commensurable de paramètre $A=a_{G} \sqrt{3}$ (fig5a); dans le composé "pauvre" il n'est pas ordonné (fig $5 \mathrm{~b}$ ).

Les figures $6 \mathrm{a}$ et $6 \mathrm{~b}$ correspondent à la rangée 101 du sous-réseau graphitique dans les deux composés; le nombre de réflexions et leurs intensités diffèrent totalement. Ces distributions d'intensité sont compatibles avec, pour $\mathrm{LiC}_{12}$, une succession des feuillets de type $\mathrm{A} / \mathrm{AA} / \mathrm{A}$..., avec $c=I_{c}$, et pour $\mathrm{Li} C_{18}$, une séquence de type $A / A B / B A / A \ldots d e$ paramètre $c=2 I_{c}$.

L'analyse de l'intensité de l'ensemble des réflexions $(\mathrm{hkl})$, il résulte que:

- dans le composé $\mathrm{LiC}_{12}$, le lithium est ordonné 3D pour former un réseau commensurable avec le graphite $\sqrt{3} \sqrt{3} \pm R 30^{\circ}$ de paramètres $\mathrm{a}=428,7 \mathrm{pm}, \mathrm{c}=702,05 \pm 0.5$ pm; l'empilement des feuillets est de type AA $\alpha \mathrm{AA} \alpha \mathrm{A} . .$. , le lithium n'occupant qu'un seul site $\alpha$, avec $\mathrm{di}_{(\mathrm{AA})}=324,5 \mathrm{pm}$ et $\mathrm{di}_{(\mathrm{AaA})}=378 \mathrm{pm}$;

- dans le composé $\mathrm{LiC}_{18}$, le lithium n'est pas organisé, il occupe les puits de potentiel du graphite de façon aléatoire, la maille du réseau carboné a pour paramètres $a=247,25 \mathrm{pm}$, $c=2 \mathrm{Ic}=706,5 \times 2 \mathrm{pm}$ et l'empilement des feuillets est de type $\mathrm{A} / \mathrm{AB} / \mathrm{BA} / \mathrm{A} \ldots$ ou $\mathrm{di}_{(\mathrm{AB})}=333 \mathrm{pm}$ et $\mathrm{di}_{(\mathrm{A} / \mathrm{A})}=373,5 \mathrm{pm}$.

\section{Références}

[1] F.Saldi, M.Lelaurain and D.Billaud, Sol.St.Comm. 80 (1991) 649-56.

[2] D.Billaud, F.X Henry and P; Willmann, Mat. Res. Bull. 28 (1993) 477.

[3] R.Gay et H.Gasparoux, Les Carbones 1, A.Pacault, Masson (1965) pp 62-128.

[4] V.A.Drits, C.Tchoubar, X-Ray Diffraction by Disordered Lamellar Structures, SpringerVerlag (1990) p9.

[5] R.Moret, Intercalation in Layered Materials,M.S.Dresselhaus, Nato (1986) pp 185-232.

[6] A.Plançon, F.Rousseaux, D.Tchoubar, C.Tchoubar, G.Krinari and V.A. Drits, J. App. Cryst. 15 (1982) 509-512.

[7]J.P.Eberhart, Analyse structurale et chimique des Matériaux, Dunod, (1989) pp201.

[8] D.Guérard and A.Herold, Carbon 13 (1975) 337-345.

[9] D.Billaud, E.Mc Rae, J.F.Marêché and A.Hérold, Synth.Met; 3 (1981) 21-26.

[10]K.C. Woo, W.A. Kamitakahara, D.P. Di Vicenso, D.G.Robinson, H.Mertwoy, J.W. Milliken and J.E.Fisher, Phy. Rev. Lett. (1983) 182-185.

[11] D.Billaud, F.X.Henry, M.Lelaurain and P.Willmann, J. Phys.Chem.Sol. à paraître 